\title{
ANTROPOLOGÍA
}

\section{CUIDAR ES ANTIGUO COMO EL MUNDO Y TAN CULTURAL COMO LA DIVERSIDAD DE LA HUMANIDAD}

\section{CECILIA ROHRBach-VIADAS}

Enfermera antropóloga, doctorado en sociología y en antropología, Universidad de Lausanne, Suiza. Docencia e investigación en instituciones interesadas en la ciencia de cuidados y cultura.

Chemin de Mâcheret 25, 1095 Lutry. Suiza.E-mail: froc@vtx.ch

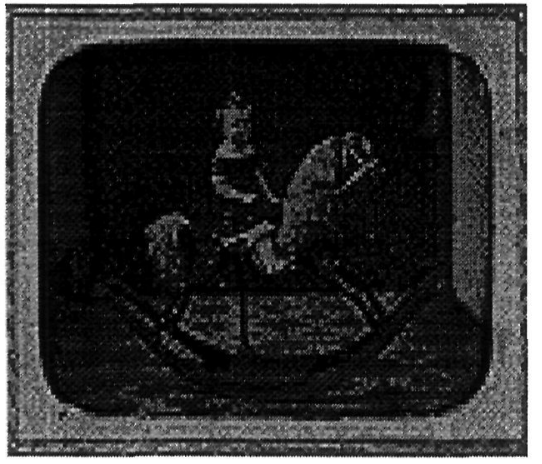

\section{RESUMEN}

Este artículo describe mi encuentro con la antropología y las experiencias que transformaron mi filosofía, mi concepción del ser humano y mi profesión de origen. La enfermería y la antropología han estado alejadas una de la otra durante poco más de un siglo y existen raíces históricas que explican este hecho, sin necesariamente justificarlo. La ciencia de los cuidados está unida a la cultura y mi propósito es introducir el interés teórico, filosófico y metodológico para los cuidados enfermeros.

Albert Bisbee

«Niño en su caballo balancín»

\section{CARING IS ANCIENT AS THE WORLD AND CULTURAL AS DIVERSITY OF HUMANITY}

\section{SUMMARY}

This article describes my encounter with Anthropology and the experiences that transformed my philosophy, my conception of human beings and my initial profession. Nursing and Anthropology have been far from each other for over a century and there are historical roots explaining such fact has without necessarly justifying it. The science of care is linked to culture and my purpose is to introduce the theoretical, philosophical and methodological interest for Nursing.

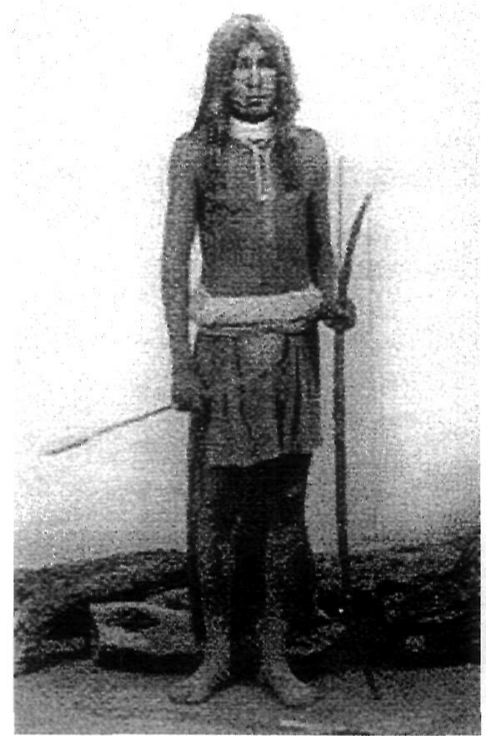




\section{a) Introducción}

Mi experiencia con los cuidados y la antropología está constituida de rodeos. Los rodeos que he realizado para descubrir al otro estudiando una cultura diferente de la mía (los campesinos mexicanos, una aldea tradicional, un grupo de tamules) ha sido una manera de comprenderme a mí misma, de avanzar conociendo la cultura del otro, haciendo comprensible la mía. Estudiar otra cultura enseña a mirar la propia de manera diferente. Comparando se descubren las diferencias. Así «cuidar y cultura significan para mí la experiencia de comprenderse uno mismo por el rodeo del otro» (Rohrbach, 1997a).

La enfermería cardiológica ha sido durante varios años mi práctica clínica y es la enseñanza experiencial la que forma la mayor parte de mi experiencia docente. $\mathrm{Mi}$ interés por la pedagogía me hizo realizar una maestría en ciencias de la educación en la Sorbona y más tarde preparar el doctorado ya mencionado. Mi primer curso de antropología me convenció que esos conocimientos y los ritos de rigor, como el año de trabajo de campo en una aldea que yo escogí tradicional y campesina (San José de Gracia, Michoacán, México), era lo que yo buscaba para completar mi formación universitaria.

\section{b) Cuidar y la perspectiva cultural}

Fue el trabajo de campo con la comunidad campesina (Rohrbach, 1992) la que ha dado más significado a mi reflexión, el contacto diario durante un año con una cultura desconocida para mí ha sido una experiencia pedagógica insustituible. Descubrí una nueva concepción del ser humano que me desconcertó y que aprendí a respetar profundamente. Esa concepción que me enseñaron los campesinos, tiende a la igualdad y al reparto de riquezas. Los campesions practican relaciones simétricas y de redistribución en su comunidad, dan una importancia particular a los lazos sociales y a la alianza social de compadrazgo, porque es vital para ellos en ciertos momentos de la vida y en las crisis (Rohrbach, 1992: 169-184). Los campesinos tienen una manera de cuidar la vida y la práctica de las relaciones simétricas están asociadas a su identidad. ¿Para qué es útil a la enfermera tener esos conocimientos?

Suponiendo que la enfermera cuidase a esta comunidad y se dedicara al grupo de los ancianos. Esto significaría para los otros campesinos, que la enfermera estaría privilegiando a un grupo y no a los otros y este hecho traería problemas, ya que el grupo campesino sentiría una ofensa hacia su propia identidad y buscaría la manera de defenderse (Rohrbach, op. cit.).

Los campesinos (en este caso un grupo de 66 habitantes), no aceptan los privilegios porque éstos rompen la igualdad y las personas que vienen del exterior reciben el mismo tratamiento. Si la enfermera sabe y comprende esta situación cultural en la que ella va a cuidar una comunidad, buscará la manera que el grupo se sienta respetado y reconocido como grupo y como individuos. Su actitud no puede ser un comportamiento ya que se trata de un proceso cambiante que hay que integrar y equilibrar continuamente.

Estudiar la cultura y los cuidados abren cuidar a un campo nuevo, espacioso, en el que desafíos constantes fuerzan a la creatividad, que estimula la reflexión, porque cuidar es también ayudar al otro a ser independiente y para saber cómo, es indispensable descubrir su modo de vivir; su teoría del mundo.

Vivir con los campesinos favorece el aprendizaje porque el proyecto de cuidar es mutuo, yo no puedo construir una realidad sola, es necesario dejarla emerger con ellos.

\section{c) Cuidar en enfermería}

Cuidar es el deseo de toda estudiante de enfermería y de toda enfermera profesional. Pero, ¿qué sabemos sobre cuidar? ¿qué se nos enseñó sobre cuidar? Cuidar, desde el punto de vista de la formación quiere decir que la enfermera se forma, aprende un modelo, lo aplica. Aprende una cultura que es esencialmente científica y médica. La primera escuela de formación profesional y laica en el mundo en cuidados de enfermería es la de «La Source» en Lausanne, fundada por Madame Valérie de Gasparin (Jaccard, 1949, p. 358, et La Source, 1859-1959, citada por Nadot, 1992: 334). El programa de Florence Nightingale propone un programa parecido al anterior y basado también en el modelo médico (cursos de anatomía y concimientos médico-quirúrgicos) en el Hôpital de St. Thomas de Londres en 1860, el cual es el segundo en el mundo.

El primer siglo de enseñanza de la enfermería ha sido científico, cuantitativo y positivista. Ha buscado esencialmente el perfeccionamiento tecnológico. La medicina científica ha contribuido a la imagen de la profesión. Habitualmente se ve a la enfermera con una jeringa lista para picar, o la enfermera misma utiliza el estetoscopio alrededor del cuello como reconocimiento profesional. La enfermería marcada por la técnología médica, por la enfermedad, por la farmacología, por los medios de diagnóstico para curar se ha valorizado asociando estos valores al prestigio propio. Desde 1955-1960 han empezado cambios en la profesión, como la preocupación por la relación con el paciente introduciendo nuevas ideas y valores humanistas que empezaron a reconocerse como propios. Los cuidados directos al paciente, la significación de cuidar, el poco reconocimiento de la enfermería como profesión, el salario inferior pagado a la enfermera, el alto porcentaje de mujeres en la profesión son to- 
dos elementos que forman una corriente fuerte contra la que hay que luchar para reconocer y hacer reconocer el concepto de cuidar.

Cuidar es aún un fenómeno poco conocido y poco estudiado, durante más de un siglo se ha asimilado cuidar al hospital hasta el punto de hablar de «extrahospitalario». Se ha centrado cuidar en actos aprendidos, en técnicas. Esos cuidados aprendidos en la escuela se oponen al cuidar que la humanidad ha inventado desde que la vida existe para que ésta misma se desarrolle. Cuidar empieza por uno mismo y está íntimamente relacionado con las propias y previas experiencias con los cuidados y con la cultura en la que se nace y en la que se vive. Cuidar se experimenta desde pequeño con el contacto de la madre que cuida. Se crece en un medio donde se cuida de una cierta manera y también se estudia o se trabaja en un ambiente en el que se pone esmero y se desarrolla el cuidado. En ese respecto, la experiencia previa de cuidar influye sobre la capacidad misma de hacerlo. La práctica de cuidar hace realizar los conocimientos necesarios a la ciencia de cuidar con una perspectiva cultural que ya tiene treinta años de experiencia (Leininger, M. 1970). En todo enfoque de cuidar tomando en consideración la cultura y el ser humano, se asocian; y es importante señalar aquí que la mayoría de las enfermeras que han elaborado un modelo teórico de enfermería (Peplau, Roy, Orem, Henderson, etc.) han descrito una concepción del hombre universalista, sin cultura. Es desde luego importante tener una concepción universal primero, sin embargo, hoy en día la cultura subrayada por la importancia que ha tomado la identidad cultural, necesita estar presente en toda reflexión teórica sobre cuidar. Una razón que puede explicar porque no ha sido el caso, es que la enfermería es aún una profesión etnocéntrica y esto ha detenido el progreso de los conocimientos sobre la cultura, la posibilidad de realizar experiencias y estudios de campo. Cómo explicar que sólo dos enfermeras tienen un doctorado en antropología en Europa 1997: Helle Ploug Hansen (agosto 1992) de Dinamarca y Cecilia Rohrbach (enero 1992) de Suiza.

\section{d) Breve reflexión sobre el origen de cuidar}

Los seres humanos de diferentes culturas del mundo han cuidado y siguen cuidando la mayor parte del tiempo la vida desde que ésta nace. Este hecho es el verdadero origen de cuidar, ya que cuidar es tan viejo como el mundo y tan cultural como la diversidad que caracteriza a la humanidad. Esa realidad acentúa la necesidad de estudiar y de enseñar las ciencias humanas en toda formación de enfermería y particularmente la antropología. Además de los cursos de antropología se necesita formarse en la ciencia de los cuidados y la cultura que es diferente de la antropología. El trabajo de Leininger es ejemplar en ese sentido. Estudiar los cuidados de otras , culturas pertenece a nuestra profesión porque la humani- ! dad es diversa y no podemos pretender que los cuidados de una cultura sean válidos para todas las demás. Esto sería prueba de etnocentrismo. Cada cultura tiene sus prácticas, sus creencias, sus valores, sus tradiciones y éstas son tan válidas e importantes como las occidentales.

\section{e) Principios filosóficos que guían la investigación y la práctica de los cuidados dentro de la perspectiva cultural}

Decubrir una cultura diferente de la propia implica una reflexión filosófica sobre los valores que guían el estudio. La ética aprendida en la escuela de enfermería es frecuentemente insuficiente cuando se trata de costumbres que generalmente son repudiadas por nuestra sociedad. Por ejemplo, la ablación del clítoris en ciertas comunidades africanas, las visitas en gran número cuando un paciente gitano es hospitalizado. ¿Cómo guiar la práctica? ¿Sobre que principios basarse?

Dos principios filosóficos guían mi reflexión, mi investigación con las otras culturas:

1) Respetar la igualdad de las culturas.

2) Reconocer las diferencias culturales (Rohrbach, 1997b).

Esos dos principios contienen para mí las diferentes situaciones de cuidados y cultura que pueden presentarse. Lo complicado es el trabajo constante de vigilarse uno mismo para observar la aplicación de esos principios considerados como «la cuadratura del círculo» en el medio de la antropología crítica suiza (Berthoud, 1992: 126).

Cuidar es una ontología: una manera de ser, una manera de mirar, de pensar, de compartir y de participar con el otro y de describir la experiencia que permite al mismo tiempo el conocimiento propio. Cuidar es un fenómeno social y cultural, una ciencia social y humana que necesita preparación y conocimientos y sobretodo experiencia de trabajo de campo. Para cuidar a pacientes de otras culturas hay que desarrollar el esmero de cuidar universalmente, de sentirse capaz de identificarse a cualquier ser humano porque es humano. Se cuida el patrimonio de la humanidad cuando se aprende a cuidar, a respetar y a reconocer a los seres humanos de cada cultura incluyendo la propia. Los pacientes de la propia cultura son cuidados de manera uniforme, como se ha enseñado durante la formación y esta mirada cambia cuando descubre uno la importancia de la cultura del otro.

\section{f) Una experiencia de campo ¿Por qué y cómo?}

Es cierto que no es posible conocer todas las culturas del mundo, pero es posible realizar una o varias expe- 
riencias pedágogicas para descubrir una o varias culturas o grupos culturales, para interiorizar la experiencia de la diferencia y aprender a hacer emerger los valores, las creencias, las tradiciones del grupo estudiado. Para descubrir la cultura se dispone de otro paradigma para salir del individualismo y descubrir un grupo. Se trata de descubrir la cultura que no es ni fija, ni está siempre en movimiento: la cultura que se ha trasmitido desde varias generaciones y la cultura que se adquiere, la cultura que da significado a todo lo que nos rodea, que da el sentimiento tan necesario para el ser humano de pertenecer a un lugar, a una familia, a un grupo. Esta experiencia de campo trae con ella otra mirada sobre cuidar con una perspectiva cultural (Rohrbach, 1997c).

\section{Epílogo}

La enfermería moderna y la antropología han caminado por direcciones contrarias. La enfermería se modeló en gran parte con la medicina, la higiene, las leyes naturales, lo observable y los procedimientos cuantitativos. La antropología recorrió el mundo para estudiar a los seres humanos de esa «familia humana» que empezó a existir científicamente desde el siglo XIX. Así, los antropólogos trataron de describir las sociedades tradicionales y completar el estudio del ser humano del que queda aún tanto por descubrir. En ese estudio del ser humano, hay un blanco casi total en lo que respecta al estudio de cuidar y de la cultura. Nuestras colegas americanas (Leininger, 1970; Spector, 1996; Morse, 1989; Brink, 1989; Aamodt, 1989) han avanzado, pero en Europa este estudio está aún por desarrollarse. Europa tiene una cultura que le es propia como continente y tiene una diversidad cultural marcada que enriquece ese mismo continente. Las formaciones europeas y las americanas son diferentes $y$ por eso podemos enriquecernos unos a otros, pero hay que recordar la importancia de la identidad europea y afirmar una trayectoria y una posición teórica, metodológica y epistemológica. Cuidar y cultura constituyen la ciencia de la enfermera(o) sin que eso quiera decir que se tiene el monopolio de cuidar. Cuidar es universal y no distingue ni cultura, ni raza, ni edad, ni grupo profesional. Es propio a todo ser humano, aunque existen innumerables diferencias culturales. Sin embargo, la ciencia de cuidar pertenece a un grupo profesional, a la enfermería. Desarrollar su investigación y estudio es la mejor manera de reatrapar nuestro retraso con lo humano caracterizado por la cultura. Vivimos en un siglo multicultural y el siglo que viene lo será aún más. No se perderá la identidad cultural sino que probablemente se afirmará (Leininger: comunicación personal, Preiswerk y Sauvain-Dugerdil, 1993). Mi responsabilidad profesional es de favorecer el cuidado del patrimonio humano; de los seres que lo constituyen a través de la investigación y de la docen- cia, y el amor de lo simple de cuidar, que tanta falta hace en nuestro medio.

\section{BIBLIOGRAFÍA}

Berthoud, G. (1992) Vers une anthropologie générale. Librairie Droz, Paris, Genève.

Hansen, H. P. (1994) Information and levels of communication-an ethnographic investigation of cancer nursing. Journal of Cancer Care 3, 18-21.

Kilani, M. (1994) L'invention de l'autre. Éditions Payot, Lausanne.

Leininger, M. (1970) Nursing and anthropology: Two worlds to blend. John Wiley \& Sons, New York.

Leininger, M. (1991) Culture Care Diversity and Universality: A Theory of Nursing. National League of Nursing, New york.

Leininger, M. (1994) Nursing and Anthropology: Two Worlds to Blend. Greyden Press, (2nd Edition), Ohio.

Leininger, M. (1995) Transcultural Nursing. Concepts, Theories, Research \& Practices. McGraw Hill, (2nd Edition), New York..

Morse, J. (1989) Qualitative Nursing Research. A Contemporary Dialogue. Sage Publications, London.

Nadot, M. (1992) Des «Médiologues» de santé à Fribourg? Thèse de doctorat, Uni. Lumière - Lyon 2.

Preiswerk, Y. y Sauvain-Dugerdil, C. (1993) Vers un ailleurs prometteur... L'émigration, une réponse universelle à une situation de crise? P.U.F. et Cahiers de l'IUED, Paris, Genève.

Rohrbach, C. (1992) Regard autre sur la vieillesse. Éditions Payot, Lausanne.

Rohrbach, C. (1997a) Philosophical Reflections on Diversity and Universality. A paraître: Journal of Nursing Science. Conférence donnée au 23 e Symposium de Recherche en Soins Infirmiers Transculturels, Université de Kuopio, Finlande.

Rohrbach, C. (1997b) Soigner c'est l'expérience de se comprendre soi-même par le détour de l'autre. A paraître.

Rohrbach, C. (1997c) «Comment la culture donne des multiples significations à "prendre soin"?» Actes du IV Colloque du Réseau Multinational de Recherche et de Réflexion á partir de la pratique Quotidienne des soins infirmiers (PRAQSI), Portugal.

Spector, R. (1996) Cultural Diversity in Health and Illness. Appleton \& Lange, Stamford. 